\title{
Mode of Conception and Risk of Spontaneous vs. Provider-Initiated Preterm Birth: Population-based cohort study
}

\author{
Yimin Wang ${ }^{1}$, Joel Ray ${ }^{2}$, Jessica Pudwell ${ }^{1}$, Laura Gaudet ${ }^{1}$, Yingwei Peng ${ }^{1}$, and Maria \\ Velez $^{1}$ \\ ${ }^{1}$ Queen's University \\ ${ }^{2}$ Departments of Medicine and Obstetrics and Gynaecology, St. Michael's Hospital, \\ University of Toronto, Ontario, Canada
}

February 11, 2022

\begin{abstract}
Objective: To study the association between mode of conception and risk of preterm birth (PTB), including, spontaneous and provide-initiated PTB. Design: Retrospective cohort study Setting and Population: Population-based cohort included all singleton livebirths and stillbirth in Ontario, Canada, 2006-2014. Methods: Mode of conception comprised (i) subfertility without infertility treatment $(\mathrm{N}=68,822)$; (ii) non-invasive infertility treatment (ovulation induction $+/$ - intrauterine insemination) ( $\mathrm{N}$ $=9024$ ); or (iii) Invasive infertility treatment (in vitro fertilization $[\mathrm{N}=8038$ ) - each compared to births by unassisted conception $(\mathrm{N}=646,926)$. Modified Poisson regression generated risk ratios $(\mathrm{RR})$. Confounding was handled by inverse probability of treatment weighting using a propensity that included maternal demographics and pre-existing conditions. Main Outcome Measure: PTB < 37 completed weeks' gestation. PTB was further categorized as spontaneous PTB or provider-initiated PTB. Results: PTB occurred among $6.0 \%$ of births by unassisted conception, $7.7 \%$ with subfertility, $8.0 \%$ with non-invasive infertility treatment, and $10.8 \%$ following invasive infertility treatment. The RR of provider-initiated PTB was higher in women with subfertility (RR 1.23, 95\% CI 1.16-1.31), non-invasive infertility treatment (RR 1.48, 1.29-1.69) and invasive infertility treatment (RR 2.35, 2.09-2.64) - each relative to births by unassisted conception. The corresponding RR for spontaneous PTB were 1.15 (95\% CI 1.10-1.19), 1.19 (95\% CI 1.09-1.31) and 1.40 (95\% CI 1.27-1.53). Conclusions: Subfertility, and receipt of infertility treatment, are each associated with a higher risk of PTB, especially provider-initiated PTB. Strategies are needed to reduce the underlying indications to deliver these women before term.
\end{abstract}

\section{Introduction}

Preterm birth (PTB) is a leading cause of neonatal morbidity and mortality. ${ }^{1}$ The rate of PTB has increased in many locations, ${ }^{1,2}$ predominantly because of increasing rates of provider-initiated PTB and increased access to Infertility treatment associated with high rates of multiple pregnancy. ${ }^{1}$ Nonetheless, singleton pregnancy after infertility treatment is also associated with PTB. ${ }^{3}$ It is unclear whether the baseline infertility diagnosis, type of infertility treatment, ${ }^{4}$ or pregnancy complications associated with infertility treatment, ${ }^{5}$ contribute differentially to the increased risk of PTB. ${ }^{6}$ Furthermore, others have reported that infertility without infertility treatment (i.e subfertility), may be associated with PTB. ${ }^{7,8}$

PTB is not comprised of a single entity, but different subtypes. ${ }^{1}$ About $30-35 \%$ of PTB are providerinitiated — otherwise termed "iatrogenic" or "indicated" — with rates as high as 50\% in many high-income countries. ${ }^{1,9}$ Provider-initiated PTB occurs through induction of labour or prelabour caesarean delivery, typically owing to a maternal and or fetal indication. ${ }^{1}$ The other $50 \%-70 \%$ of PTB are spontaneous, after onset of spontaneous preterm labour or preterm premature rupture of the membranes (PPROM). ${ }^{1}$ Provider-initiated 
PTB is associated with twice the risk of neonatal mortality and morbidity compared with spontaneous PTB. ${ }^{2,10,11}$

Most studies examining PTB in relation to mode of conception have assessed PTB as a single entity. There are a lack of data about the role of subfertility and non-invasive infertility treatment (Ovulation induction $[\mathrm{OI}]$, or Intrauterine Insemination [IUI]) on PTB subtypes. Studies have reported an increased risk of spontaneous PTB following Invasive- infertility treatment, namely, In VitroFertilization (IVF) and Intracytoplasmic Sperm Injection (ICSI). ${ }^{12}$ Risk factors for both spontaneous and provider-initiated PTB include non-Caucasian ethnicity, previous preterm birth, and lower socioeconomic status, among others. ${ }^{13}$ Access to infertility treatment is influenced by sociodemographic characteristics, including ethnicity, income, education, smoking and higher body mass index (BMI). ${ }^{14-16}$ To account for these factors, previous studies used matching or stratification. ${ }^{12}$ Of note, propensity score methods have more recently been used to reduce the effects of confounding in observational studies that aim to estimate treatment effects. ${ }^{17}$

Understanding the association between PTB subtypes in women with subfertility and those who receive infertility treatment may help improve strategies for PTB prevention. Accordingly, this population-based cohort study evaluated the association between mode of conception and risk of PTB, including, spontaneous and provide-initiated PTB.

\section{Methods}

\section{Study Design and Data Sources}

This population-based cohort study comprised all singleton hospital livebirths and stillbirths in Ontario, April 1, 2006 to March 31, 2014. We obtained pregnancy and neonatal outcomes from the Better Outcomes Registry \& Network (BORN) Ontario and Niday Legacy data sets (www.bornontario.ca/en/data/datadictionary/legacy-datasets/). BORN captures more than $99 \%$ of hospital births in the province and has been previously validated for completeness and accuracy. ${ }^{18}$ Excluded were pregnancies that resulted in termination, or a gestational age below 20 complete weeks. Analyses were restricted to women aged 18 to 60 years. Maternal demographics and pre-existing health conditions were obtained from the Canadian Institute for Health Information Discharge Abstract Database (CIHI DAD), National Ambulatory Care Reporting System (NACRS), Ontario Health Insurance Plan Claims Database (OHIP), Registered Persons Database (RPDB), Postal Code Conversion File (PCCF), Linked Delivering Mothers and Newborns (MOMBABY), Ontario Hypertension Dataset (HYPER), Ontario Diabetes Dataset (ODD), and the Immigration, Refugees and Citizenship Canada Permanent Resident (IRCC-PR) database - all existing linked datasets at ICES (www.ices.on.ca). ICES is an independent, non-profit research institute funded by an annual grant from the Ontario Ministry of Health $(\mathrm{MOH})$ and the Ministry of Long-Term Care (MLTC). As a prescribed entity under Ontario's privacy legislation, ICES is authorized to collect and use health care data for the purposes of health system analysis, evaluation and decision support. Secure access to these data is governed by policies and procedures that are approved by the Information and Privacy Commissioner of Ontario.

\section{Infertility treatment}

The exposure of interest was the mode of conception recorded in BORN, namely, i) unassisted conception (the reference group); ii) subfertility without infertility treatment (i.e., a history of an infertility consult with a physician, defined as an ICD-9 628 OHIP diagnosis in the 2 years prior to the estimated date of conception, and in the absence of receipt of infertility treatment); iii) non-invasive infertility treatment (i.e., ovulation induction [OI] or intrauterine insemination [IUI] alone); and iv) invasive infertility treatment (i.e., In Vitro fertilization [IVF] or intracytoplasmic sperm injection [ICSI]).

\section{Preterm birth subtypes}

The primary study outcome was PTB $<37$ completed weeks' gestation. PTB was further categorized as spontaneous PTB or provider-initiated PTB. ${ }^{1}$ Births that result from spontaneous preterm labour and PPROM were classified as spontaneous PTB, while preterm birth that resulted from labour induction or 
caesarean delivery for maternal or fetal indications were classified as provider-initiated PTB. As a secondary outcome, we assessed the outcome of early PTB $<34$ weeks' gestation.

\section{Statistical analysis}

Descriptive statistics summarized the proportion of PTB, overall and by subtypes according to mode of conception. To study the effect of confounding by indication of treatment and fertility status, inverse probability of treatment weighting (IPTW) using propensity score was applied. ${ }^{17,19}$ The weights were derived to obtain estimates representing the average treatment effect for the treated (ATT). ${ }^{17}$ The underlying propensity score model included maternal age; obesity (i.e., pre-pregnancy BMI $>30 \mathrm{~kg} / \mathrm{m}^{2}$, or, if BMI was missing from BORN, we used the OHIP billing code for obesity [ICD-9 278); parity; smoking; pre-pregnancy and gestational diabetes mellitus; chronic hypertension; immigration; rurality; and income quintile; along with the statistical interaction of parity with age, smoking with obesity, history of preterm birth with age, history of preterm birth with diabetes, and income quintile with age (Table S1). The balance between treatment population was evaluated by standardised differences of baseline covariates, using a threshold of $>0.1$ signifying important differences. ${ }^{20}$

The relation between mode of conception and PTB was quantified by absolute rates and risk ratios (RR), derived using modified Poisson regression with a robust error variance, which also accounts for correlated errors among potentially more than one birth occurring in the same woman, ${ }^{21}$ and adjusted for baselines characteristics selected through ATT weights. ${ }^{17}$

This study was reviewed for ethical compliance by the Queen's University Health Sciences \& Affiliated Teaching Hospitals Research Ethics Board and received initial clearance on October 29, 2019 (Reference number is 6028050). Data were analyzed using SAS software version 9.1 (SAS Institute, Cary, NC, 2010).

\section{Results}

A total of 732,810 singleton livebirth and stillbirths were included, among 649,918 women. Of these, 646,926 (88.3\%) were by unassisted conception; $68,822(9.4 \%)$ in patients with a history of subfertility; $9024(1.2 \%)$ after non-invasive infertility treatment, and 8038 (1.1\%) following invasive infertility treatment (Figure 1). Patients with subfertility, non-invasive infertility and invasive infertility treatment were more likely to be older, nulliparous, obese, and more likely to have diabetes. Chronic hypertension was more common in women with non-invasive and invasive infertility treatment (Table 1).

IPTW achieved a balance in the confounding variables in the weighted sample (Figure S1). There were 45,343 cases of PTB $<37$ weeks' gestation $(6.2 \%)$, of which 33,117 (4.5\%) were spontaneous and 12,226 $(1.7 \%)$ provider-initiated. Moreover, these figures translate into $73 \%$ of all PTB being spontaneous and $27 \%$ provider-initiated, further differing depending on the mode of conception (Figure 2). For example, while provider-initiated PTB accounted for $26 \%$ of all PTB in women with spontaneous conception (i.e., $10,033 / 38,444)$, it contributed to $40 \%$ of all PTB in those with invasive infertility treatment (i.e., 350/869).

Relative to births by unassisted conception, the adjusted RR of PTB $<37$ weeks was increased in women with subfertility, non-invasive and invasive infertility treatment (Figure 2, top). The adjusted RR of providerinitiated PTB $<37$ weeks was higher in women with subfertility (1.23, 95\% CI 1.16-1.31), non-invasive infertility treatment $(1.48,1.29-1.69)$ and invasive infertility treatment $(2.35,2.09-2.64)$ - each relative to births by unassisted conception (Figure 2). The corresponding adjusted RR for spontaneous PTB $<37$ weeks were 1.15 (95\% CI 1.10-1.19), 1.19 (95\% CI 1.09-1.31) and 1.40 (95\% CI 1.27-1.53) (Figure 2).

For the outcome of PTB $<34$ weeks' gestation, the RRs followed a similar pattern as for PTB $<37$ weeks (Figure 2). One exception was among women with subfertility, who were not at appreciably higher risk of provider-initiated PTB < 34 weeks' gestation (adjusted RR 1.08, 95\% CI 0.95-1.23).

\section{Discussion}

Main Findings 
In this population-based study, PTB was more likely to occur in women with subfertility or those undergoing infertility treatment. With more invasive infertility treatment, the RR of spontaneous PTB, and particularly, provider-initiated PTB, was highest.

\section{Strengths and Limitations}

This study used a large population-based sample from a validated provincial registry, comprising all hospital births, within a publicly funded health care system. The ability to categorize women by type of conception, including subfertility and type of infertility treatment is another strength. One limitation could be nondifferential misclassification of the exposure: Subfertility was based on a single OHIP billing code of ICD-9-628 in the 2 years prior to the index conception date, in the absence of receipt of infertility treatment during that time. In those who conceived by infertility treatment, this OHIP billing code appeared among $90 \%$ of women with non-invasive infertility treatment, and $96 \%$ of those with invasive infertility treatment, indirectly suggesting that clinically subfertile women were being captured herein. On the other hand, exposure to infertility treatment was captured by chart review by trained abstractors at the time of birth, and is likely to be accurate as are other data elements in the Ontario birth registry. ${ }^{22}$ However, it is possible that some pregnant individuals who conceived by non-invasive or invasive infertility treatment were not captured by this approach resulting in nondifferential misclassification and a possible underestimate of the effect on PTB. In fact, capturing infertility and fertility treatment status from birth certificate records has been found to be highly specific yet poorly sensitive, suggesting that while some females with infertility are missed with this approach, false positives are unlikely. ${ }^{23}$

\section{Interpretation}

The overall rate of PTB in our study is consistent with that reported in Canada (6.3 per 100 singleton livebirths) ${ }^{24}$ Our estimates of PTB are also in agreement with the Massachusetts Outcomes Study of Assisted Reproductive Technologies (MOSART), ${ }^{8}$ which reported an OR of PTB of 1.24 (95\% CI 1.21, 1.38) in patients with subfertility, and 1.53 (95\% CI 1.40-1.67) following Invasive infertility treatment. The MOSART study did not provide information on non-invasive infertility treatment, or detail PTB subtypes. Moreover, in a recent meta-analysis of 15 studies, comprising 61,677 singleton births, and which reported a pooled OR of 1.63 (95\% CI 1.30-2.05) for spontaneous PTB after IVF/ICSI, the risk provider-initiated PTB was not assessed therein. ${ }^{12}$ In a population-based study of 1813 sibling pairs in the Netherlands Perinatal Registry, no association was found between IVF and either spontaneous or provider-initiated PTB, compared to unassisted-conceived siblings. ${ }^{25}$

The overall contribution of provider-initiated PTB to all PTB in our study (27\%), is similar to that reported in Canada (25\%). ${ }^{9}$ However, this contribution varied in our study by mode of conception: $26 \%$ after unassisted conception and $40 \%$ after invasive infertility treatment. One-third of provider-initiated PTB are associated with preeclampsia and/or intrauterine growth restriction, and this proportion increases with earlier gestational age at birth. ${ }^{9}$ Assisted Reproductive technology (ART), including IVF, ICSI, oocyte donation and frozen embryo transfer, is associated with an increased risk of the hypertensive disorders of pregnancy, ${ }^{5}$ and fetal growth restriction ${ }^{4}$ - both established reasons for provider-initiated PTB. Subfertility and infertility treatment too are associated with an increased risk of prelabour cesarean delivery, both at term and preterm. ${ }^{26}$

Among women with subfertility, or those undergoing infertility treatment, strategies are needed to lessen their risk of provider-initiated PTB, while minimizing maternal and neonatal morbidity. Care plans for women pregnant after ART are few. ${ }^{27}$ In early pregnancy, a simple approach is to use available clinical risk factors, including ART, that readily identify women at risk for preeclampsia. ${ }^{28}$ Level 1 evidence shows that low-dose aspirin modestly reduces the risk of spontaneous PTB, small for gestational age, birthweight, and maternal preeclampsia. ${ }^{29}$ Future studies should consider the benefit of low-dose aspirin starting at 12 weeks' gestation in women with subfertility, or those undergoing IT, who are at higher risk for preeclampsia and PTB.

\section{Conclusion}


Among singleton pregnancies, subfertility and infertility treatment were each associated with an increased risk of PTB, especially for provider-initiated PTB. Future studies should focus on interventions or strategies that can reduce the risk for provider-initiated PTB in these women.

\section{Disclosure of interests}

None declared.

\section{Contribution to authorship}

YPW: statistical analysis, interpretation of the data, drafting of manuscript. MV: Study concept, interpretation of the data, manuscript revision, and approval of final version. JGR, JP, LG, YPP: interpretation of the data, manuscript revision, and approval of final version.

\section{Details of Ethics Approval}

This study was reviewed for ethical compliance by the Queen's University Health Sciences \& Affiliated Teaching Hospitals Research Ethics Board.

\section{Funding}

This study was made possible with funding from the Canadian Institutes of Health Research (CIHR) Institute of Human Development, Child \& Youth Health (IHDCYH), Clinician-Investigator Teams in Obstetrics and Maternal-Fetal Medicine, Grant number MFM - 146444.

\section{Acknowledgements}

This study was supported by ICES, which is funded by an annual grant from the Ontario Ministry of Health and Long-Term Care (MOHLTC). No endorsement by ICES or the Ontario MOHLTC is intended or should be inferred. Parts of this material are based on data and/or information compiled and provided by CIHI. However, the analyses, conclusions, opinions and statements expressed in the material are those of the author(s), and not necessarily those of CIHI. This study is based, in part, on data provided by the Better Outcomes Registry \& Network (BORN), part of the Children's Hospital of Eastern Ontario. The interpretation and conclusions contained herein do not necessarily represent those of BORN Ontario. Parts or whole of this material are based on data and/or information compiled and provided by Immigration, Refugees and Citizenship Canada (IRCC) current to March 31st, 2014. However, the analyses, conclusions, opinions and statements expressed in the material are those of the authors, and not necessarily those of IRCC. The authors would like to acknowledge Paul Nguyen at ICES Queen's for assistance with cohort creation.

\section{Data sharing}

The data set from this study is held securely in coded form at ICES. While data-sharing agreements prohibit ICES from making the data set publicly available, access may be granted to those who meet prespecified criteria for confidential access, available at www.ices.on.ca/DAS. The full data set creation plan and underlying analytic code are available from the authors upon request, understanding that the computer programmes may rely upon coding templates or macros that are unique to ICES and therefore either inaccessible or requiring modification.

\section{References}

1. Goldenberg RL, Culhane JF, Iams JD, Romero R. Epidemiology and causes of preterm birth. Lancet. 2008;371(9606):75-84.

2. Barros FC, Velez Mdel P. Temporal trends of preterm birth subtypes and neonatal outcomes. Obstet Gynecol. 2006;107(5):1035-1041.

3. McDonald SD, Han Z, Mulla S, et al. Preterm birth and low birth weight among in vitro fertilization singletons: a systematic review and meta-analyses. Eur J Obstet Gynecol Reprod Biol.2009;146(2):138-148. 
4. Elias FTS, Weber-Adrian D, Pudwell J, et al. Neonatal outcomes in singleton pregnancies conceived by fresh or frozen embryo transfer compared to spontaneous conceptions: a systematic review and meta-analysis. Arch Gynecol Obstet. 2020;302(1):31-45.

5. Chih HJ, Elias FTS, Gaudet L, Velez MP. Assisted reproductive technology and hypertensive disorders of pregnancy: systematic review and meta-analyses. BMC Pregnancy Childbirth. 2021;21(1):449.

6. Shapiro-Mendoza CK, Lackritz EM. Epidemiology of late and moderate preterm birth. Semin Fetal Neonatal Med. 2012;17(3):120-125.

7. Basso O, Baird DD. Infertility and preterm delivery, birthweight, and Caesarean section: a study within the Danish National Birth Cohort.Hum Reprod. 2003;18(11):2478-2484.

8. Declercq E, Luke B, Belanoff C, et al. Perinatal outcomes associated with assisted reproductive technology: the Massachusetts Outcomes Study of Assisted Reproductive Technologies (MOSART). Fertil Steril.2015;103(4):888-895.

9. Ray JG, Bartsch E, Park AL, Shah PS, Dzakpasu S. Estimated reductions in provider-initiated preterm births and hospital length of stay under a universal acetylsalicylic acid prophylaxis strategy: a retrospective cohort study. CMAJ Open. 2017;5(2):E508-E516.

10. Chen A, Feresu SA, Barsoom MJ. Heterogeneity of preterm birth subtypes in relation to neonatal death. Obstet Gynecol.2009;114(3):516-522.

11. Lisonkova S, Hutcheon JA, Joseph KS. Temporal trends in neonatal outcomes following iatrogenic preterm delivery. BMC Pregnancy Childbirth. 2011;11:39.

12. Cavoretto P, Candiani M, Giorgione V, et al. Risk of spontaneous preterm birth in singleton pregnancies conceived after IVF/ICSI treatment: meta-analysis of cohort studies. Ultrasound Obstet Gynecol. 2018;51(1):43-53.

13. Berkowitz GS, Blackmore-Prince C, Lapinski RH, Savitz DA. Risk factors for preterm birth subtypes. Epidemiology.1998;9(3):279-285.

14. Jain T. Socioeconomic and racial disparities among infertility patients seeking care. Fertil Steril. 2006;85(4):876-881.

15. Farland LV, Collier AY, Correia KF, et al. Who receives a medical evaluation for infertility in the United States? Fertil Steril.2016;105(5):1274-1280.

16. Moreau C, Bouyer J, Ducot B, Spira A, Slama R. When do involuntarily infertile couples choose to seek medical help? Fertil Steril.2010;93(3):737-744.

17. Austin PC. An Introduction to Propensity Score Methods for Reducing the Effects of Confounding in Observational Studies. Multivariate Behav Res. 2011;46(3):399-424.

18. Arbuckle TE, Liang CL, Morisset AS, et al. Maternal and fetal exposure to cadmium, lead, manganese and mercury: The MIREC study. Chemosphere. 2016;163:270-282.

19. Xu S, Ross C, Raebel MA, Shetterly S, Blanchette C, Smith D. Use of stabilized inverse propensity scores as weights to directly estimate relative risk and its confidence intervals. Value in health : the journal of the International Society for Pharmacoeconomics and Outcomes Research. 2010;13(2):273-277.

20. Austin PC. Balance diagnostics for comparing the distribution of baseline covariates between treatment groups in propensity-score matched samples. Stat Med. 2009;28(25):3083-3107.

21. Zou G. A Modified Poisson Regression Approach to Prospective Studies with Binary Data. American Journal of Epidemiology.2004;159(7):702-706. 
22. Dunn S, Lanes A, Sprague AE, et al. Data accuracy in the Ontario birth Registry: a chart re-abstraction study. BMC Health Serv Res. 2019;19(1):1001.

23. Zhang Z, Macaluso M, Cohen B, et al. Accuracy of assisted reproductive technology information on the Massachusetts birth certificate, 1997-2000. Fertil Steril. 2010;94(5):1657-1661.

24. Public Health Agency of Canada. (2017). Perinatal Health Indicators for Canada 2017: A Report from the Canadian Perinatal Surveillance System.

25. Seggers J, Pontesilli M, Ravelli ACJ, et al. Effects of in vitro fertilization and maternal characteristics on perinatal outcomes: a population-based study using siblings. Fertil Steril.2016;105(3):590-598 e592.

26. Richmond E, Ray JG, Pudwell J, et al. Caesarean birth in women with infertility: population-based cohort study. BJOG. 2021.

27. Velez MP, Hamel C, Hutton B, et al. Care plans for women pregnant using assisted reproductive technologies: a systematic review.Reprod Health. 2019;16(1):9.

28. Bartsch E, Medcalf KE, Park AL, Ray JG, High Risk of Pre-eclampsia Identification G. Clinical risk factors for pre-eclampsia determined in early pregnancy: systematic review and meta-analysis of large cohort studies. BMJ. 2016;353:i1753.

29. Ray JG, Park AL, Fell DB. Mortality in Infants Affected by Preterm Birth and Severe Small-forGestational Age Birth Weight.Pediatrics. 2017;140(6).

\section{Figure legends}

\section{Figure Number legend}

Figure 1. Study flow chart

Figure 2. Mode of conception and risk of preterm birth (PTB), provider-initiated PTB and spontaneous PTB. R

Figure S1. Standardized mean differences between unassisted conception and overall group of other conception mo

\section{Table legends}

\section{Table Number legend}

Table $1 . \quad$ Characteristics of 732,810 singleton hospital births by mode of conception before propensity score weigh Table S1. Variables included in the propensity score model selection

Hosted file

Table 1.docx available at https://authorea.com/users/460053/articles/556132-mode-ofconception-and-risk-of-spontaneous-vs-provider-initiated-preterm-birth-population-basedcohort-study

\section{Hosted file}

Figure 1.docx available at https://authorea.com/users/460053/articles/556132-mode-ofconception-and-risk-of-spontaneous-vs-provider-initiated-preterm-birth-population-basedcohort-study

\section{Hosted file}

Figure 2.docx available at https://authorea.com/users/460053/articles/556132-mode-ofconception-and-risk-of-spontaneous-vs-provider-initiated-preterm-birth-population-basedcohort-study 